\title{
Surgical technique for atrial-esophageal fistula repair after catheter ablation: An underrecognized complication
}

Brandon A. Guenthart, MD, ${ }^{a}$ Beatrice Sun, MD, ${ }^{\mathrm{b}}$ Andreas De Biasi, MD, ${ }^{\mathrm{a}}$ Michael P. Fischbein, MD, PhD, ${ }^{\mathrm{a}}$ and Douglas Z. Liou, MD, ${ }^{a}$ Stanford, Calif

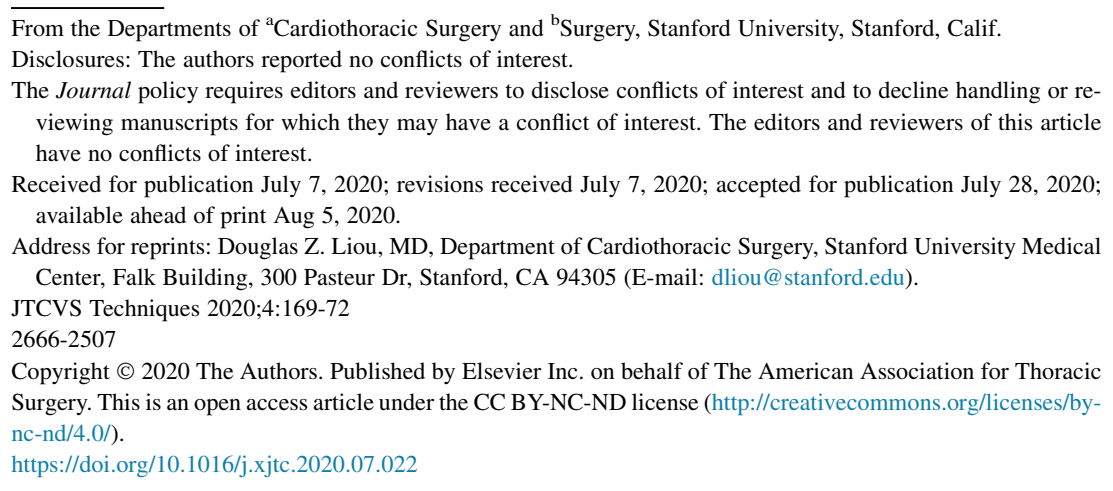

Video clip is available online.

Atrial-esophageal fistula (AEF) is a rare but devastating complication after catheter ablation for atrial fibrillation (AF), with mortality up to $80 \%{ }^{1}$ Prompt diagnosis and surgical intervention are essential. Herein, we describe a 2-stage approach focused on minimizing risk of air embolus and stroke, starting with intracardiac repair on cardiopulmonary bypass (CPB) followed by fistula takedown and esophageal repair via right thoracotomy. Additional novel aspects of our technique include the use of prone imaging following oral contrast administration to aid in diagnosis and the use of a stapling device during fistula takedown.

\section{CLINICAL SUMMARY}

The authors obtained signed informed consent from the patient to publish this manuscript. A 35-year-old man who underwent pulmonary vein isolation and catheter ablation for AF presented 3 weeks following the procedure with pleuritic chest pain, shortness of breath, and blurry vision. Computed tomography scan revealed oral contrast extravasation from the esophagus to the left atrium (LA) (Figure 1, $A$ and $B$ ). He was transferred emergently to our institution for immediate repair (Video 1).

The first stage of the procedure started with median sternotomy and aortic/bicaval cannulation for CPB. Endoscopy was not done initially out of concern for causing air embolism. After we arrested the heart, a left atriotomy was

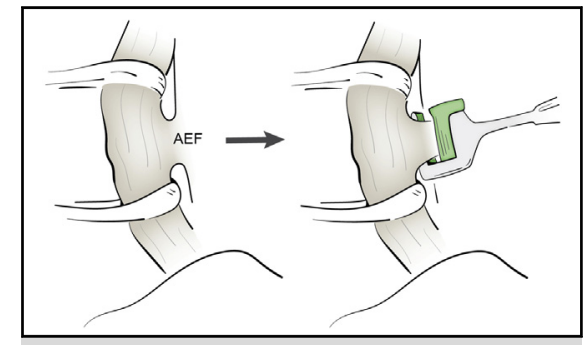

Successful surgical management of atrialesophageal fistula (AEF) with 2-staged repair.

\section{CENTRAL MESSAGE \\ Catheter-ablation procedures \\ for atrial fibrillation are \\ increasing. Life-threatening \\ complications, such as fistula \\ development, require early \\ recognition, diagnosis, and sur- \\ gical intervention.}

See Commentaries on pages 173,175 , and 176.

performed in Sondergaard's groove. The posterior wall was found to be thickened, inflamed, and densely adherent to the pericardium. Two defects in the myocardium were identified (Figure 1, C). Intracardiac repair was performed using a bovine pericardium patch to cover and exclude the defects. The patient was then separated from bypass, the sternotomy incision closed, and he was repositioned for right thoracotomy to perform stage 2 .

The fistula was isolated (Figure 2,A) and taken down with a $30-\mathrm{mm}$ TA stapler green cartridge (Figure 2, B). The esophageal defect was repaired in layers (Figure 2, $C)$ and buttressed with an intercostal muscle flap (Figure 2, D). Endoscopic leak test confirmed an intact repair; however, a contained leak was seen on esophagram the following week. An esophageal stent and jejunostomy tube were placed, and the patient was discharged home on postoperative day 14. In addition, blood cultures drawn on admission grew Lactobacillus, necessitating a prolonged course of antibiotics. On postoperative day 40 , the stent was removed and his esophagram was normal. He resumed a regular diet with no sequelae related to his swallowing. 

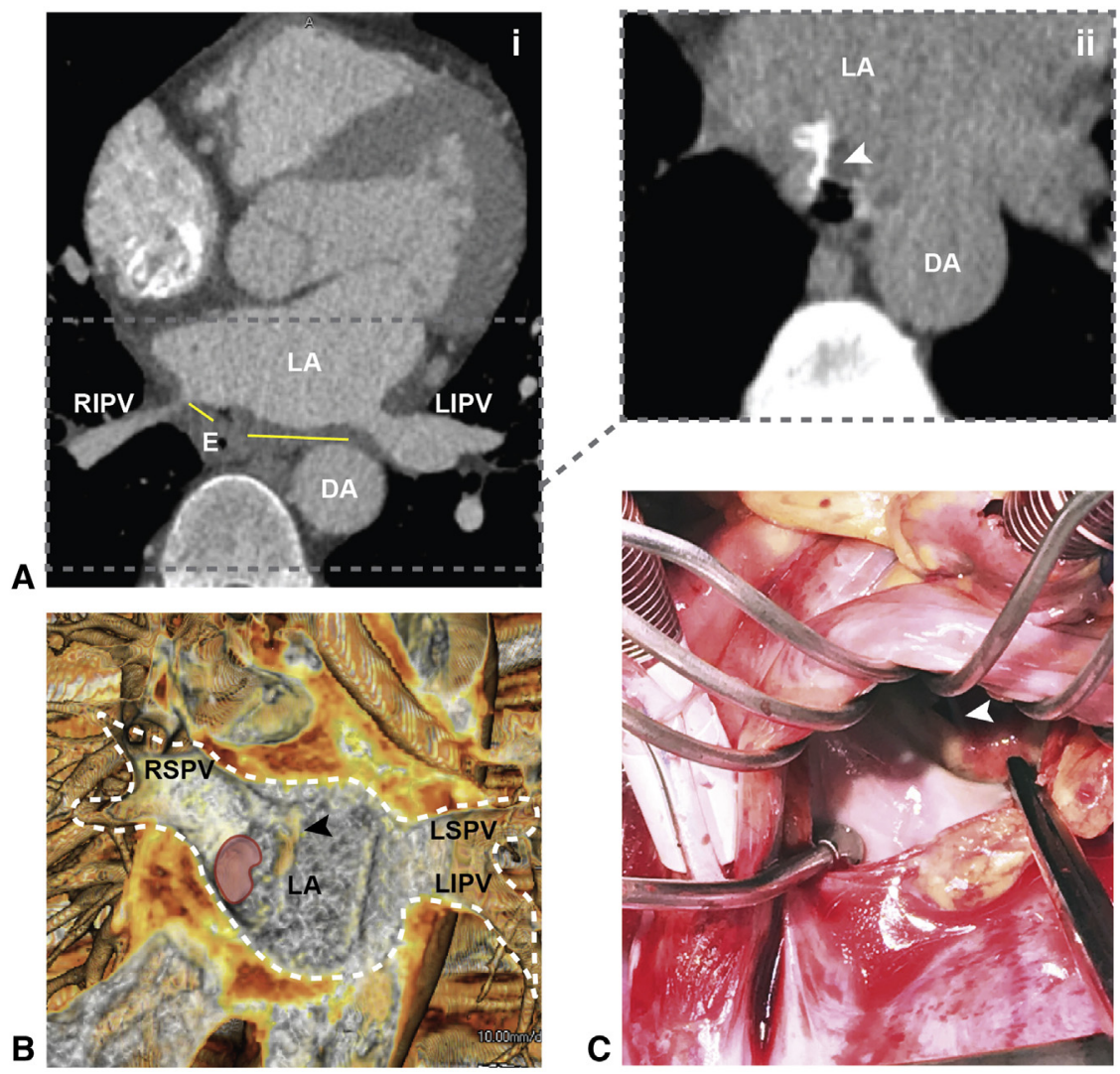

FIGURE 1. Diagnosis and initial control of atrial-esophageal fistula via median sternotomy (stage 1): A 35-year-old man underwent pulmonary vein isolation and catheter ablation for AF. Three weeks following the procedure, he presented with chest pain and blurry vision. A, Diagnostic imaging including: (i) Computed tomography with intravenous contrast (yellow line represents distance from esophagus to the ostia of the pulmonary veins) and (ii) oral contrast given and images obtained with patient lying prone demonstrating contrast extravasation and air within the atrium (white arrow). B, Three-dimensional reconstruction of the left atrium (dotted white line) demonstrating air behind the atrium (black arrowhead) and atrial defect (highlighted in red). C, Initial stage of surgical repair was performed via median sternotomy and aortic/bicaval cannulation for cardiopulmonary bypass. Intraoperative gross photography highlighting the defect located in the posterior left atrial wall with surrounding inflammation (white arrowhead). Intracardiac repair was performed using a bovine pericardium patch, and the patient was separated from bypass. RIPV, Right inferior pulmonary vein; $E$, esophagus; $L A$, left atrium; $D A$, descending aorta; $L I P V$, left inferior pulmonary vein; $R S P V$, right superior pulmonary vein; $L S P V$, left superior pulmonary vein.

\section{DISCUSSION}

The LA is often enlarged and thinned in AF, making the adjacent esophagus prone to thermal injury during ablation procedures. Recognition of esophageal injury (erythema, ulceration, perforation) following ablative therapies is increasing, leading many to advocate for increased use of postprocedural endoscopy or endoscopic ultrasonography. ${ }^{2}$ AEFs carry a high rate of mortality and are likely underreported due to a multitude of factors, including death outside the hospital, delay in diagnosis, and misdiagnosis. As symptoms can be vague and time to development of AEF is broad (days to months), clinicians must be cognizant of all possible complications following ablative therapy and have a low threshold for investigation.

The most common imaging modality to diagnose AEFs is computed tomography with oral and/or intravenous contrast. $^{3}$ We recommend administering oral contrast

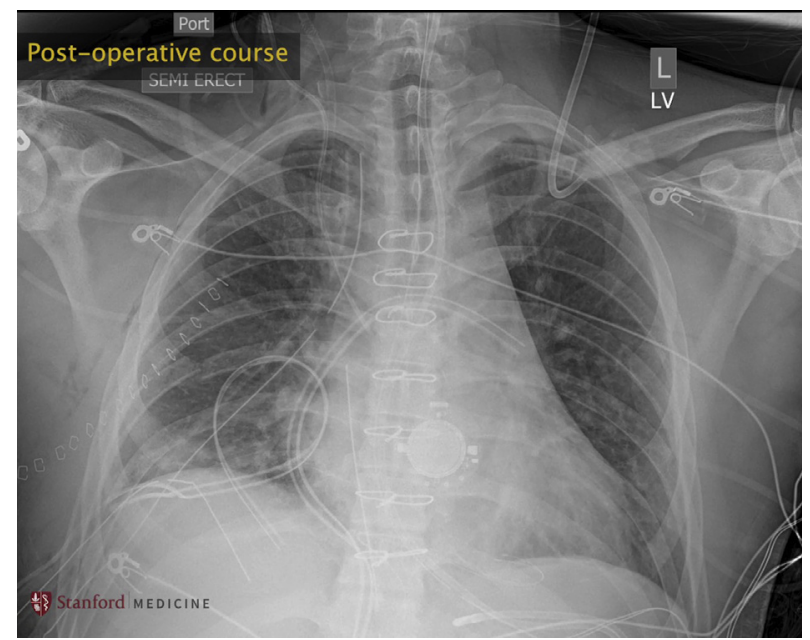

VIDEO 1. Case details including workup, surgical approach, and techniques presented in a male with an atrial-esophageal fistula following catheter ablation. Video available at: https://www.jtcvs.org/article/S26662507(20)30360-6/fulltext. 

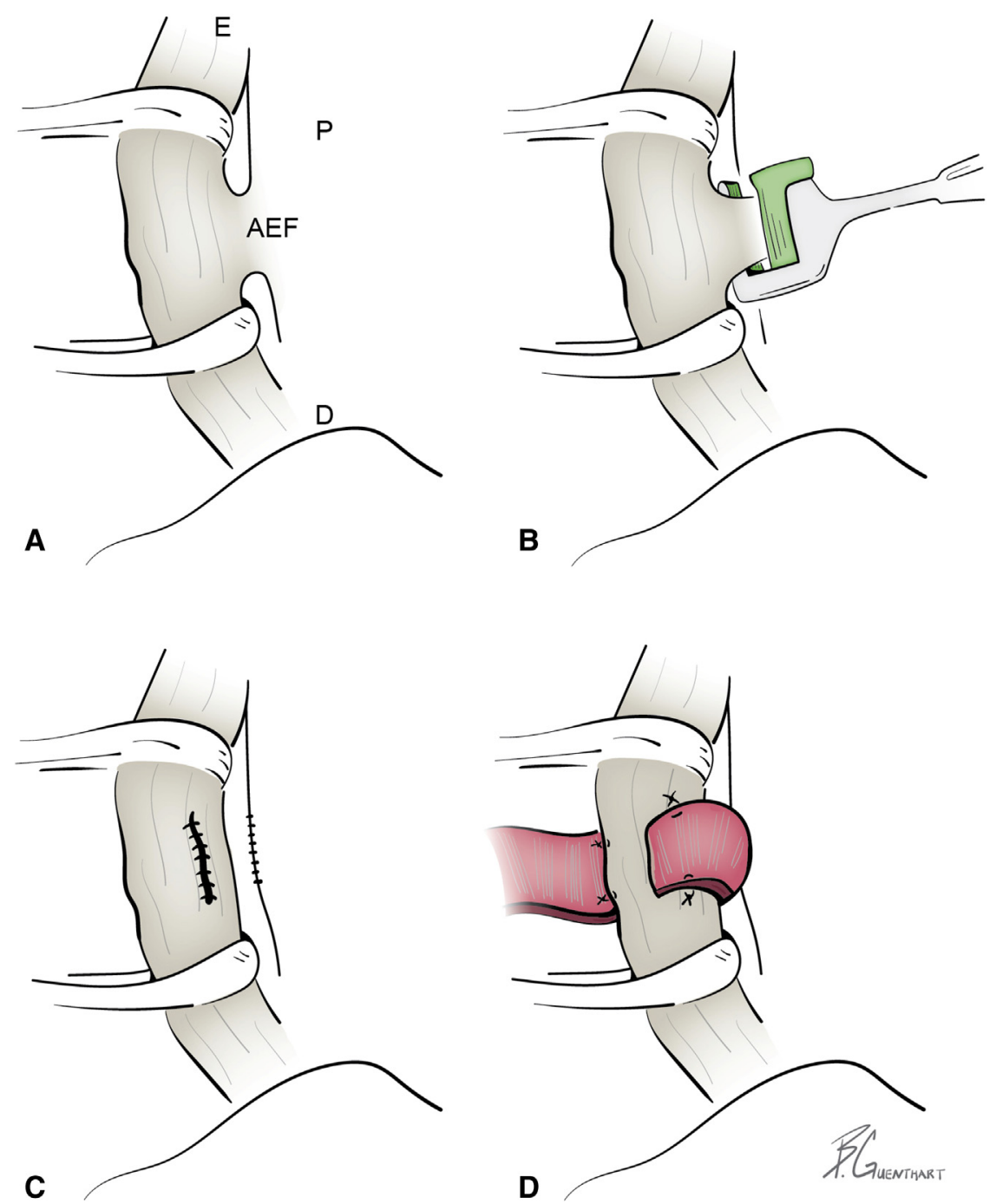

FIGURE 2. Takedown and repair of atrial-esophageal fistula via right posterolateral thoracotomy (stage 2). Following intracardiac repair via median sternotomy, the patient was repositioned and a right posterolateral thoracotomy was performed. Diagram depicts surgical steps, including A, dissection of the esophagus and identification of the atrial-esophageal fistula. Penrose drains placed along proximal and distal portions of the esophagus to aid in exposure. B, Division of esophageal fistula using TA 30-mm green $(4.8 \mathrm{~mm})$ staple load. Staple line placed close to the esophagus to avoid disruption of the pericardium and before intracardiac repair. C, Repair of the esophageal defect in layers. D, Intercostal muscle flap (harvested on entry) placed between the pericardium and esophageal repair, secured with four horizontal mattress 4-0 absorbable VICRYL sutures. The repair was then evaluated with intraoperative endoscopy and a leak test was negative. Up until this point, endoscopy was completely avoided and the patient was maintained in slight Trendelenburg to decrease the risk of cerebral air embolism. $E$, Esophagus; $P$, pericardium; $A E F$, atrial-esophageal fistula; $D$, diaphragm.

immediately before image capture and with the patient in prone position, as was done in this case. Once diagnosed, treatment includes broad-spectrum antibiotics and immediate surgical intervention. Nonoperative management and endoscopic therapies alone are inferior and associated with unacceptably high mortality.

Cerebral air embolism often results in devastating neurologic sequelae. Air can enter the systemic circulation from the esophagus during either endoscopy or CPB until the fistula has been taken down. Several strategies were used in this case to minimize the risk of this dreaded complication.
The patient was maintained in slight Trendelenburg, expeditiously placed on CPB with early crossclamp application, and upper endoscopy was completely avoided until the fistula was divided, as there are reports of deteriorating neurologic status and death following insufflation from endoscopy. ${ }^{4}$

Although various surgical approaches have been described, we advocate a staged 2-field technique (median sternotomy and right thoracotomy) using CPB. In doing so, the LA is decompressed and intracardiac repair is completed before esophageal repair to prevent embolic 
neurologic injury. This approach (intracardiac repair first with $\mathrm{CPB}$ ) also protects against massive hemorrhage with fistula manipulation.

Following takedown of the AEF and esophageal repair in layers, the esophagus should be buttressed with muscle (intercostal, latissimus, pectoralis) and/or fat (pericardial, omental flap). This reinforces the repair while also providing added physical separation between the inflamed esophagus and heart. Diversion with cervical esophagostomy should be performed in the setting of hemodynamic instability or friable esophageal tissue not suitable for repair. Feeding access can be considered at the time of the index procedure or placed in a delayed fashion if needed. In the setting of uncontrolled leak or a large defect, return to the operating room for esophageal diversion is likely necessary.

As the incidence of catheter ablation procedures for $\mathrm{AF}$ is increasing worldwide, ${ }^{5}$ complications associated with the procedure are becoming more relevant. In the case of lifethreating AEFs, early recognition, diagnosis, and surgical intervention is imperative and associated with improved survival.

\section{References}

1. Gray WH, Fleischman F, Cunningham MJ, Kim AW, Baker CJ, Starnes VA, et al. Optimal approach for repair of left atrial-esophageal fistula complicating radiofrequency ablation. Ann Thorac Surg. 2018;105:e229-31.

2. Zhang P, Zhang Y-Y, Ye Q, Jiang RH, Liu Q, Ye Y, et al. Characteristics of atrial fibrillation patients suffering esophageal injury caused by ablation for atrial fibrillation. Sci Rep. 2020;10:1-9.

3. Ha FJ, Han HC, Sanders P, Teh AW, O'Donnell D, Farouque O, et al. Challenges and limitations in the diagnosis of atrioesophageal fistula. J Cardiovasc Electrophysiol. 2018;29:861-71.

4. French K, Garcia C, Wold J, Hoesch R, Ledyard H. Cerebral air emboli with atrialesophageal fistula following atrial fibrillation ablation: a case report and review. Neurohospitalist. 2011;1:128-32.

5. Han H-C, Ha FJ, Sanders P, Spencer R, Teh AW, O'Donnell D, et al. Atrioesophageal fistula: clinical presentation, procedural characteristics, diagnostic investigations, and treatment outcomes. Circ Arrhythm Electrophysiol. 2017;10: e005579. 